\title{
EFECTO DE RECUBRIMIENTOS DE ALMIDÓN MODIFICADO DE YUCA, PROTEINA AISLADA DE SOYA Y ACEITE ESENCIAL DE ORÉGANO APLICADOS A LA PAPAYA
}

\section{EFFECT OF MODIFIED YUCA STARCH COATINGS, ISOLATED PROTEIN OF SOYA AND OREGANO ESSENTIAL OIL APPLIED TO THE PAPAYA}

\author{
Dora Alejandra Castillo Silva ${ }^{1}$, Katterine Salazar Anacona ${ }^{2}$, Silvio Andrés Mosquera Sánchez ${ }^{3}$, Edwuin Rengifo Canizales ${ }^{4}$
}

${ }^{1}$ Ingeniera Agroindustrial. Universidad del Cauca, Transversal 9A No.60N-172, Popayán-Cauca, Colombia, e-mail: alejac26@ hotmail.com; ${ }^{2}$ Ingeniera Agroindustrial. Universidad del Cauca, Carrera 9N No. 67N-14, Popayán-Cauca, Colombia, e-mail: katherine_salazar16@hotmail.com; ${ }^{3}$ Magíster en Ingeniería, profesor titular. Universidad del Cauca, Calle 35N No. 21-200, Popayán-Cauca, Colombia, e-mail: smosquera@unicauca.edu.co; ${ }^{4}$ Magíster en Ingeniería de Sistemas. Universidad del Cauca, Calle 5 No. 4-70, Popayán-Cauca, Colombia, e-mail: erengifo@unicauca.edu.co

\author{
Rev. U.D.C.A. Act. \& Div. Cient.21(1): 71-80, Enero-Junio, 2018 \\ https://doi.org/10.31910/rudca.v21.n1.2018.664
}

\section{RESUMEN}

La papaya tiene elevada demanda en el mundo; sin embargo, la producción y la exportación se afectan por microorganismos fitopatógenos y pérdidas pos cosecha, cercanas al $25-40 \%$ en la cadena de suministro, sobre todo, en transporte y en almacenamiento, debido a un deficiente manejo. La aplicación de recubrimientos comestibles permite mejorar el brillo y la textura de la corteza, reducir el deterioro de la calidad fisicoquímica y organoléptica, la pérdida de peso por deshidratación y el intercambio de gases. Por lo anterior, durante trece días, en papayas recubiertas con almidón de yuca modificado variedad SM 707-17 (4\%), proteína aislada de soya (2\%) y aceite esencial de orégano (250ppm y 500ppm), se evaluó el efecto sobre pérdida de peso, de color, de firmeza, de sólidos solubles, de respiración, de $\mathrm{pH}$, de acidez titulable y de crecimiento def Fusarium spp., a condiciones ambientales, mediante un diseño completamente al azar con dos factores, tiempo y tratamientos. El experimento, se hizo por triplicado y los datos fueron sometidos a un análisis estadístico, utilizando el programa SPSS V.23. Los resultados indicaron que la utilización de almidón, por sí solo o combinado con aceite esencial de orégano (250ppm) y proteína aislada, lograron reducir la pérdida de peso, controlar la respiración al reducir el porcentaje de $\mathrm{CO}_{2}$ y retardar el desarrollo del color amarillo y rojo en las papayas frente al control, además de retrasar el crecimiento del hongo Fusarium solani; también, se encontró que los recubrimientos no incidieron sobre características fisicoquímicas, como el pH, la acidez titulable, los sólidos solubles y la firmeza de los frutos.
Palabras clave: Carica papaya, Origanum vulgare, Fusarium solani, Glycine max (L.), Manihot esculenta Crantz. (Tesauro y Glosario Agrícola).

\section{SUMMARY}

The papaya has a high demand in the world, however, its production and export are affected by phytopathogenic microorganisms and post-harvest losses close to $25-40 \%$ in the supply chain, especially in transport and storage due to poor management. The application of edible coatings improves the gloss and texture of the bark, reduces the deterioration of the physicochemical and organoleptic quality, the weight loss due to dehydration, and the exchange of gases. Therefore, for thirteen days, papayas were coated with cassava starch modified SM 707-17 variety (4\%), isolated soy protein (2\%) and oregano essential oil (250ppm and 500ppm). The effect was evaluated on weight loss, color, firmness, soluble solids, respiration, $\mathrm{pH}$, titulable acidity and growth of Fusarium spp., to environmental conditions. A completely random design with two factors, time and treatments, the experiment was done in triplicate, and the data were subjected to a statistical analysis using the SPSS V.23 program. The results indicated that the use of starch alone or combined with essential oil of oregano (250ppm) and isolated protein were able to reduce weight loss, control respiration ( $\mathrm{CO}_{2}$ percent) and slow down the development of the yellow and red color in the papayas against the control in addition to delaying the growth of the fungus Fusarium spp. It was also found that the coatings did not affect physicochemical characteristics such as $\mathrm{pH}$, titulable acidity, soluble solids, and firmness of the fruits. 
Key words: Carica papaya, Origanum vulgare, Fusarium solani, Glycine max (L.), Manihot esculenta Crantz.

\section{INTRODUCCIÓN}

La papaya, se ha posicionado como un producto de importancia, a nivel mundial, debido a su aporte en antioxidantes, en vitamina $B$, en minerales (potasio, magnesio) y en fibra, además, es fuente de papaína (enzima digestiva), utilizada en industrias de cervecería, carnes, farmacéutica, productos de belleza y cosmética; sin embargo, su producción y su exportación se ha visto afectada, por su alto contenido de humedad, entre el 80 y el 85\% (Ocoró \& Ayala, 2013), el deficiente manejo durante el transporte y almacenamiento y el desarrollo de enfermedades, causadas por patógenos del género Fusarium, ampliamente conocidos, por producir metabolitos tóxicos, que ponen en peligro la salud de los seres humanos (Villa et al. 2015); como consecuencia de lo anterior, se generan pérdidas del 25 a 40\%, del total del cultivo.

Las investigaciones en poscosecha de frutas y de hortalizas permiten mejorar la conservación al aplicar tecnologías alternativas al uso de ceras comerciales, para reducir la contaminación ambiental y prolongar la vida útil de los productos. Dentro de las ceras comerciales están las que son de origen natural y las de origen sintético, como la colofonia, shellac y ceras de polietileno oxidada, derivadas del petróleo; estas últimas, de difícil degradación, lo que genera persistencia en el ambiente $y$, por ende, un impacto ambiental. Adicionalmente, la mayoría de las ceras comerciales no son digestibles, por lo que se debe pelar el fruto recubierto, antes de consumirlo. En los últimos años, el enfoque de la conservación de productos frescos ha sido el desarrollo y la investigación en polímeros degradables, un ejemplo, es el uso de recubrimientos comestibles, que se caracterizan por ser biodegradables y amigables con el ambiente, al estar constituidos por materias primas renovables, en algunos casos, extraídas de subproductos de la agroindustria. Los films preparados a partir de almidón y de proteína de soya son un claro ejemplo, al reducir el deterioro de la calidad fisicoquímica y organoléptica, la pérdida de peso y la tasa respiratoria en frutas y en hortalizas (Figueroa et al. 2013; Miranda et al. 2014; Guerreiro et al. 2015; Saavedra \& Algecira, 2010). Estos films pueden ser adicionados con agentes antimicrobianos, como los aceites esenciales utilizados para el control de enfermedades desarrolladas por hongos y por bacterias, proporcionando al alimento un mayor tiempo de vida útil (Barrera et al. 2012; Siqueira et al. 2012; Bosquez et al. 2010) y por lo general, empleados en productos mínimamente procesados, debido a que reducen los procesos oxidativos (pardeamiento enzimático), el crecimiento microbiológico y aumentan la resistencia mecánica de la fruta y de la hortaliza recubierta (De Ancos et al. 2015). En los recubrimientos, la utilización de proteínas y de polisacáridos confiere propiedades de barrera a gases, como oxígeno y dióxido de carbono (Fernández et al. 2015) y, en el caso de los lípidos, su principal función es reducir la deshidratación, mejorando las características de barrera al agua (Vázquez \& Guerrero, 2013); también, se pueden utilizar antioxidantes, antimicrobianos y otras sustancias, para destacar la calidad.

En este estudio, se utilizó aceite esencial de orégano, almidón de yuca modificado y proteína aislada de soya, para evaluar la respuesta fisiológica de la papaya recubierta con estas tres materias primas mencionadas, sobre la pérdida de peso, el color, la tasa de respiración, la firmeza, los sólidos solubles, el pH, la acidez titulable y el crecimiento del hongo Fusarium solani.

\section{MATERIALES Y MÉTODOS}

Materiales. Se usaron papayas variedad Maradol procedentes del municipio del Patía, en el Departamento del Cauca (Colombia), en grado 3 de madurez (Santamaría et al. 2009); almidón de yuca variedad SM707-17, modificado enzimáticamente hasta $12 \%$ de dextrosa equivalente, suministrado por el grupo de investigación Ciencia y Tecnología de Biomoléculas de Interés Agroindustrial (CYTIBIA), de la Facultad de Ciencias Agrarias, de la Universidad del Cauca; proteína aislada de soya marca Solae de Dupont; aceite esencial de orégano con pureza del $100 \%$, utilizado como agente antifúngico, adquirido en alkimiaesenciaslflorales.com; glicerol con $99 \%$ de pureza, utilizado como plastificante en el recubrimiento, marca Blamis Dotaciones ${ }^{\circledR}$; Tween 80 , con $99 \%$ de pureza, usado como agente emulsificante, marca Quimpo y agar papa dextrosa (PDA), con $100 \%$ de pureza, como medio de cultivo para hongos marca Dibico.

Aislamiento e identificación macroscópica y microscópica de hongos fitopatógenos. Se cortaron fragmentos de tejido necrótico y sano de papayas con distintas lesiones, se trataron con solución de hipoclorito de sodio al $1 \%$ por 3 min y se lavaron 3 veces, con agua destilada. Los cortes, se incubaron en cajas de Petri, con PDA, más 0,075\% de gentamicina, durante 8 días, a $27^{\circ} \mathrm{C}$. Se realizaron improntas de los hongos que se identificaron, teniendo en cuenta las claves taxonómicas de Barnett \& Hunter (1998), usando Microscopía Óptica de Alta Resolución (MOAR), además de observar la textura, el color, la topografía en anverso y reverso de las colonias.

Efecto del aceite esencial en el crecimiento micelial del hongo Fusarium spp. in vitro. Se aplicó la metodología de Guédez et al. (2014), realizando 5 tratamientos, con 0, 100, 200, 250 y $300 \mathrm{ppm}$, incubando las cajas a $27^{\circ} \mathrm{C}$, durante 8 días. Luego de $24 \mathrm{~h}$, se midió el diámetro de las colonias y se obtuvo el porcentaje de inhibición, así: 
\% Inhibición=(Diámetro caja Petri-Diámetro tratamiento $) /$ (Diámetro caja Petri)*100

Dónde:

Diámetro caja petri: $9 \mathrm{~cm}$

Diámetro tratamiento: diámetro de crecimiento del hongo, según el tratamiento

Limpieza y desinfección de frutos. Las papayas, se lavaron con agua potable, se sumergieron en solución de Tego 51, en concentración de 50ppm, durante 15 min y se secaron al ambiente.

Preparación y aplicación del recubrimiento. Se mezcló almidón modificado de yuca $(4 \%)$ y glicerol $(2 \%)$ en agua destilada $\left(\mathrm{pH}=7\right.$ ), en el equipo HL2 (HL2 Dworks), a $75^{\circ} \mathrm{C}$, durante $30 \mathrm{~min}$, a $400 \mathrm{rpm}$ (Paz et al. 2016), hasta gelatinización del almidón. La proteína de soya, se procesó bajo las mismas condiciones del almidón, usando agua destilada, con $\mathrm{pH}=10$, debido a su poca solubilidad en $\mathrm{pH}$ ácido cerca de su punto isoeléctrico ( $\mathrm{pH} \mathrm{4,0} \mathrm{a} \mathrm{5,5).} \mathrm{Finalmente,} \mathrm{a} \mathrm{las}$ mezclas de almidón y de proteína se les incorporó Tween 80 $(0,1 \%)$ y aceite esencial de orégano, a 250 y $500 \mathrm{mg} / \mathrm{L}$.

Las papayas, se recubrieron completamente, usando un aspersor AutoJET-1550 (AutoJET-1550, spraying systems Co., Wheaton, IL, USA), a una presión de aire de 13 bar y presión de líquido de 27 psi.

Preparación del inoculo e inoculación de los frutos. Se colocaron las esporas de Fusarium solani (dos semanas de cultivo) en agua destilada estéril, a una concentración de $1,2 \times 10^{5}$ esporas/mL y las papayas, se inocularon mediante aspersión 8 horas después de haber sido recubiertas, registrando la severidad de la enfermedad, a partir de la primera aparición de los síntomas (Ademe et al. 2013).

Medición del daño. Para determinar el grado de afectación por Fusarium, se tomó una escala general no lineal de cinco niveles (con modificaciones), en donde: $1=$ Síntomas muy leves en frutos, afectando $1 \%$ del área; $3=$ Síntomas cubriendo 5\% del área de los frutos; $5=$ Síntomas cubriendo $10 \%$ del área de los frutos; $7=$ Síntomas cubriendo $25 \%$ del área de los frutos y $9=$ Síntomas cubriendo $50 \%$ del área de los frutos. Para determinar el área afectada, se utilizó la metodología de Navarro \& Arauz (1999), con modificaciones.

Durante 13 días, cada 3 días y a la misma hora, se midieron las siguientes variables:

$\%$ Pérdida peso $=(($ Peso inicio-Peso final $) /($ Peso inicio $)) * 100$

Pérdida de peso. Se registró el peso en una balanza analítica con precisión de $0,01 \mathrm{~g}$, usando la siguiente expresión:
Color. Se determinó con el colorímetro 3nh-NR110 (3nh Brand - NR110. Shenzhen 3nh Technology CO. China), utilizando la escala CIELAB, marcando la parte más verde de los frutos y midiendo sobre ella todos los días, con el fin de registrar el cambio de color de verde a amarillo durante la evaluación.

Respiración. Las frutas, se almacenaron en bolsas plásticas selladas (una papaya por bolsa), a las que se midió el porcentaje de dióxido de carbono producido, empleando el analizador de gas Oxybaby 6.0 (Oxybaby 6.0 Witt. WITT-GASETECHNIK GmbH \& Co KG. Germany).

Firmeza. Se determinó con la Maquina universal de ensayos (Shimadzu EZ-L), a velocidad de $15 \mathrm{~mm} / \mathrm{s}$ y profundidad máxima de penetración de $10 \mathrm{~mm}$, sobre tres puntos del fruto (extremo pedicelar, zona ecuatorial y extremo apical).

Sólidos solubles totales. Con el refractómetro digital Atago PAL-1 (Atago PAL-1. ATAGO USA., Inc.), depositando una gota de jugo en el lente y haciendo la corrección de la lectura.

pH. Se extrajo el jugo de la papaya y se utilizó el pH- metro digital (pH metro 780. Polco S.A.S. Colombia), para realizar la medición.

Acidez titulable. Se titularon $10 \mathrm{~mL}$ del jugo del fruto con hidróxido de sodio $0,1 \mathrm{~N}$, expresado como porcentaje de ácido málico, así:

$$
\% \text { Acidez }=(\mathrm{NaOH}(\mathrm{mL}) * \mathrm{~N} * \mathrm{MEq} \text { ácido }) /(10 \mathrm{~mL}) * 100
$$

Crecimiento microbiano. Se midió la contaminación por el hongo Fusarium solani, según la escala numérica y verbal de la enfermedad, haciendo una comparación visual de los daños presentados en los frutos y asignándoles a cada uno un valor.

Análisis estadístico. Se aplicó un diseño completamente al azar, con dos factores y 9 tratamientos (Tabla 1). Los datos colectados, se analizaron en el programa estadístico SPSS versión 23, aplicando modelos para la normalidad (ShapiroWilk) y homogeneidad de varianzas (Estadístico de Levene). Se realizó un análisis de varianza (ANOVA) y la prueba de Tukey, para los datos que cumplieron con los supuestos anteriormente mencionados; para aquellos datos en los que no hubo normalidad ni homogeneidad de varianzas, se aplicó la prueba no paramétrica de Kruskal-Wallis y para los datos, en los cuales, no se cumplió la homogeneidad de varianzas, se aplicó la prueba estadística de Games-Howell. Finalmente, se aplicó un Anova de un factor de medidas repetidas y se realizó un análisis gráfico para porcentaje de pérdida de peso, respiración $\left(\% \mathrm{CO}_{2}\right)$ y color. 
Tabla 1. Tratamientos aplicados en el desarrollo de la investigación.

\begin{tabular}{|c|c|c|c|c|c|}
\hline Tratamiento & $\begin{array}{c}\text { Almidón de } \\
\text { yuca (\%) }\end{array}$ & $\begin{array}{c}\text { Proteína aislada } \\
\text { soya (ppm) }\end{array}$ & $\begin{array}{c}\text { Aceite esencial } \\
\text { orégano (ppm) }\end{array}$ & Glicerol (\%) & $\begin{array}{c}\text { Tween } \mathbf{8 0} \\
\mathbf{( \% )}\end{array}$ \\
\hline 1 & 0 & 0 & 0 & 0 & 0 \\
\hline 2 & 4 & 0 & 0 & 2 & 0 \\
\hline 3 & 0 & 2 & 0 & 2 & 0 \\
\hline 4 & 4 & 0 & 250 & 2 & 0,10 \\
\hline 5 & 0 & 2 & 500 & 2 & 0,10 \\
\hline 6 & 4 & 0 & 500 & 2 & 0,10 \\
\hline 7 & 0 & 2 & 250 & 2 & 0,10 \\
\hline 8 & 4 & 2 & 250 & 2 & 0,10 \\
\hline 9 & 4 & 2 & 500 & 2 & 0,10 \\
\hline
\end{tabular}

\section{RESULTADOS Y DISCUSIÓN}

Identificación macroscópica y microscópica del hongo Fusarium solani. y efecto del aceite esencial de orégano sobre el crecimiento micelial in vitro. A nivel macroscópi$\mathrm{co}$, se pudo observar que el hongo Fusarium solani presenta un micelio extenso y algodonoso, con un poco de tinte amarillo en el centro; a nivel microscópico, se observaron conidios hialinos, ligeramente curvados en los extremos puntiagudos, en forma de canoa (Barnett \& Hunter, 1998). En cuanto al porcentaje de inhibición fueron más efectivos los tratamientos T3 y T4, con un 93,33\% de inhibición, seguidos por T2, con 79,50\%; T1, con $27,9 \%$ y $0 \%$, en el testigo el día 8 , demostrando que el efecto inhibitorio del aceite esencial de orégano sobre el hongo disminuye, a concentraciones por debajo de 250ppm.

Porcentaje de pérdida de peso. El Anova de medidas repetidas indicó que el porcentaje de pérdida de peso cambia con el tiempo, dependiendo del recubrimiento usado $(p<0,05)$. La mayor pérdida de peso ocurrió en el testigo (T1), con un $13 \%$, que se aceleró a partir del día 7 , seguido de los tratamientos a base de proteína T5, T7 y T3, que perdieron $11,5,9,8$ y $8,7 \%$, respectivamente, mostrando su incidencia sobre la variable, tal como lo reportan Saavedra \& Algecira (2010), quienes indicaron que el recubrimiento que presentó menor pérdida de peso $(3,15 \%)$ fue el que contenía proteína aislada de soya, incidiendo, además, la proporción de aceite usado en la formulación, al permitir la difusión de gases y aumentar la traspiración del fruto, lo que causa una disminución significativa del peso, explicando así la diferencia entre los tratamientos $\mathrm{T} 5$ y T7, que perdieron más peso que $\mathrm{T} 3$.
En los tratamientos a base de almidón, las diferencias fueron frente al testigo (T1-T6 $=3,6 \%)$, mostrando que la incorporación del aceite esencial no incidió sobre las propiedades de barrera al vapor de agua, resultado que también fue reportado en pimentón recubierto con almidón y aceite esencial de tomillo (Ordoñez et al. 2014), considerando así que el almidón de yuca fue el que actuó como barrera semipermeable al vapor de agua y no el aceite. Lo anterior, se puede deber, a las interacciones específicas del aceite con el almidón, las cuales, quizás, no fueron lo suficientemente fuertes para interferir en la barrera a vapor de agua de este material, debido a su carácter hidrofílico; comportamiento contrario a la proteína de soya, que es afifílica, característica que le permitió unirse mejor al aceite e interferir en la barrera a vapor de agua.

Color. En las coordenadas L*, a* y b*, se observaron diferencias estadísticamente significativas en el tiempo, dependiendo del tipo de recubrimiento utilizado $(p<0,05)$. La evaluación gráfica comparativa exploratoria inicial sugiere que los recubrimientos a base de almidón parecen ejercer los mayores cambios sobre estas variables, al presentar el testigo valores de $L^{*}=61,93$, a* $=25,31$, b* $=55,57$, en comparación con el tratamiento T4, cuyos valores en las coordenadas fueron 56,51, 4,33, 46,77, respectivamente (Figuras 1 y 2), lo que se podría interpretar como que este material retarda el cambio de color en las papayas, debido a la acción del recubrimiento, que forma una atmósfera modificada, que puede influenciar cambios en productos frescos, como el color y la calidad sensorial (Oms-Oliu et al. 2008 citado por Quintero et al. 2010), al retardar la síntesis de pigmentos de color naranja y rojo (carotenoides y antocianinas) en la piel. Resultados similares fueron reportados por Castricini et al. (2012), quienes encontraron que el uso de 
almidón de yuca y el almidón de carboximetilo retardaron el cambio de color de verde a rojo, en la piel de las papayas.
Tasa de respiración (Porcentaje de $\mathrm{CO}_{2}$ ). Mediante la evaluación gráfica comparativa inicial del comportamiento de

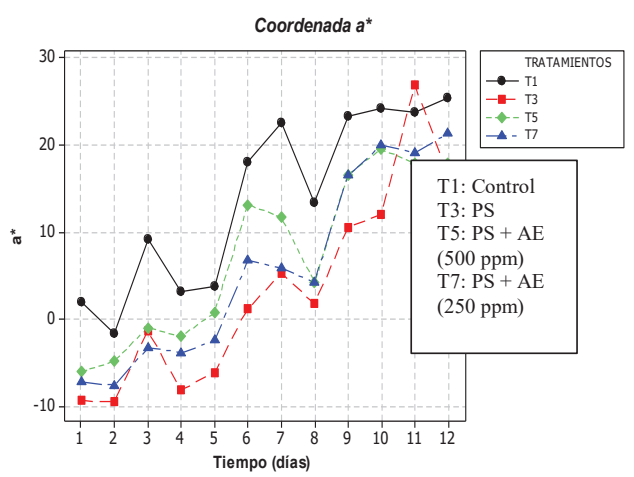

a.

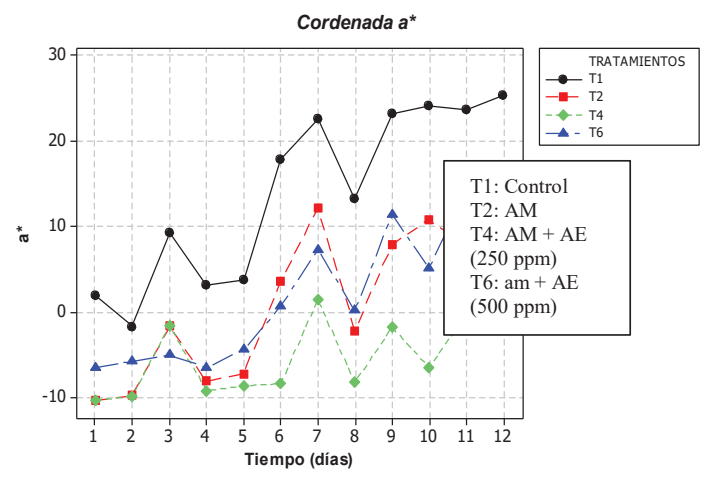

b.

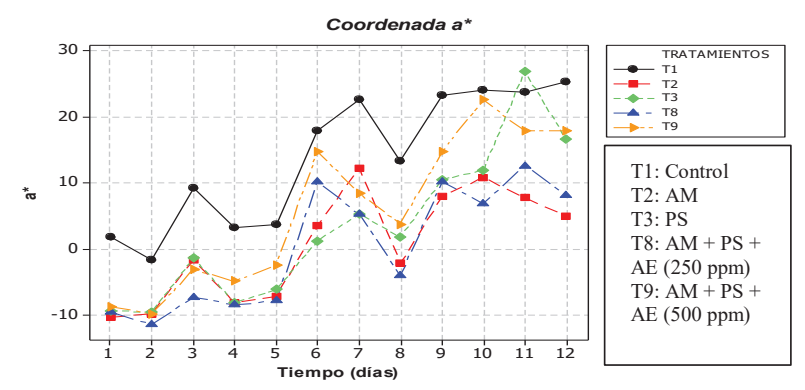

c.

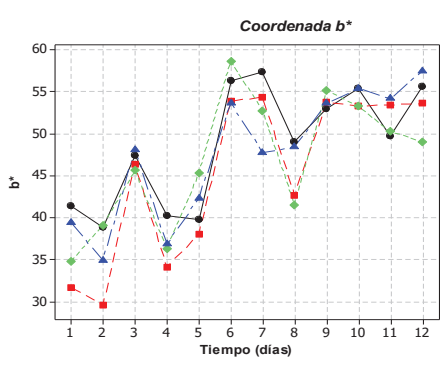

a.

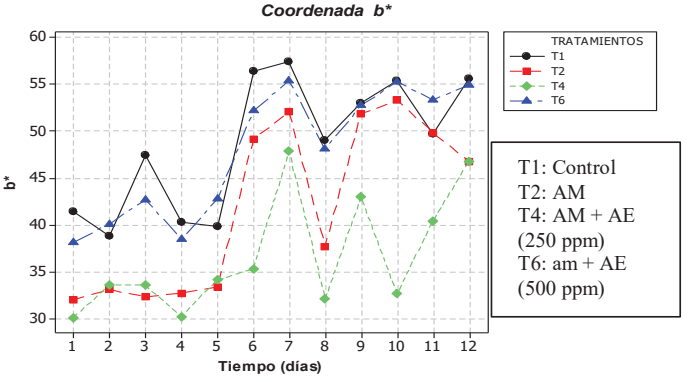

b.

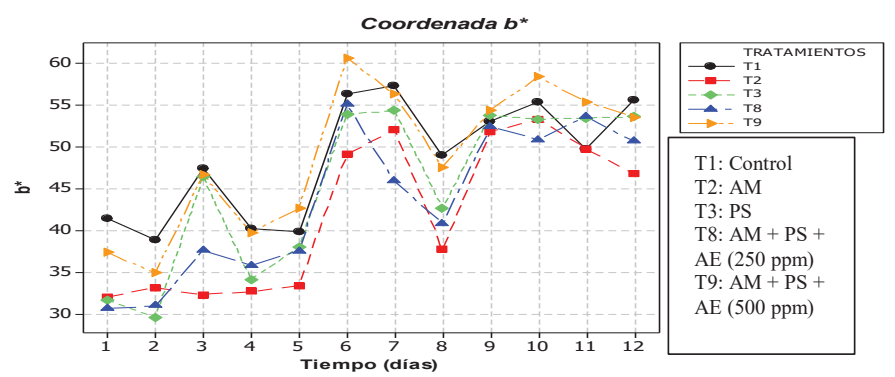

Figura 1. Cambios de color en el tiempo a 250 ppm y 500 ppm. a) Coordenadas a* y b*, para recubrimientos a base de proteína con y sin aceite esencial; b) Coordenadas a* y b*, para recubrimientos a base de almidón con y sin aceite esencial y c) Coordenadas a* y b*, para recubrimientos a base de proteína, almidón y combinados. 


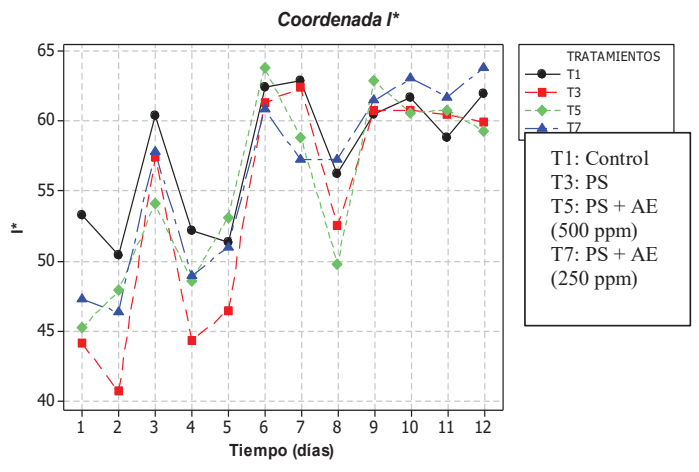

a.

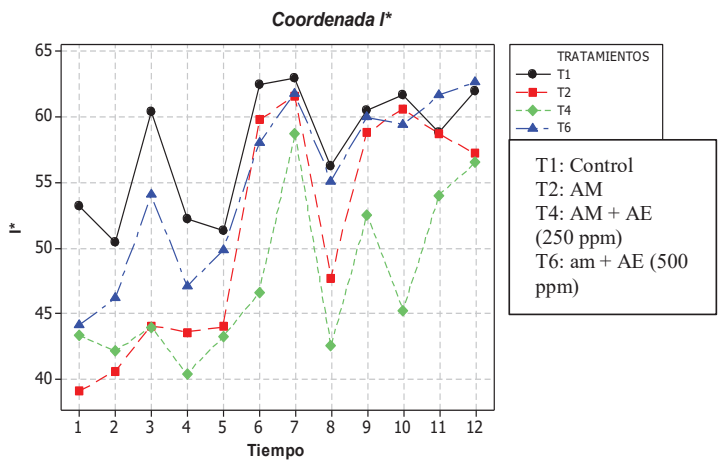

b.

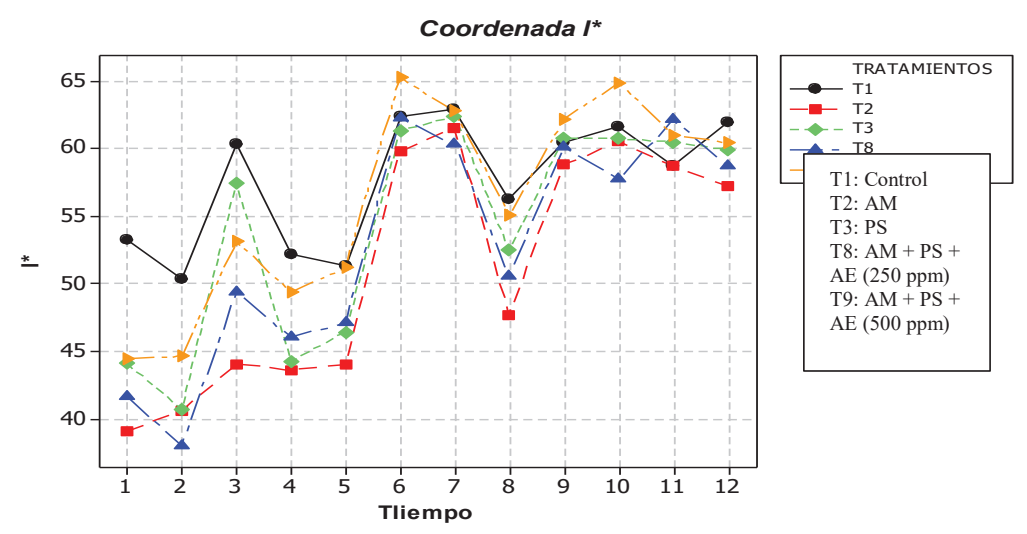

C.

Figura 2. Cambios en la coordenada I* a 250 y 500 ppm. a) Cambios en tratamientos a base de proteína con y sin aceite; b) Cambios en tratamientos a base de almidón con y sin aceite y c) Cambios en tratamientos a base de proteína, de almidón y recubrimientos combinados.

los recubrimientos en el tiempo (Figura 3), se puede observar que el máximo incremento en la producción de $\mathrm{CO}_{2}$ ocurrió a los 5 días en los frutos del control T1 (que se puede asociar con el pico climatérico). A los 7 días, se observó el pico climatérico en los tratamientos T2, T6 y T7 y, en el día 8, el del tratamiento T4, mientras que en los tratamientos T3, T5 y T9, no se observó, pues se encontró un incremento progresivo de $\mathrm{CO}_{2}$ en el tiempo, que se puede interpretar como una posible alteración del proceso respiratorio, que puede ser generado por lesiones y por enfermedades, en este caso, por el hongo Fusarium solani. El tratamiento T8 generó menores porcentajes de $\mathrm{CO}_{2}$ y menores cambios, indicando que la mezcla de almidón, de proteína de soya y de aceite esencial proporcionó una buena acción de barrera, reduciendo, así, la respiración del fruto, lo que se vio reflejado en una menor pérdida de peso (Figura 3).

Se encontraron diferencias estadísticamente significativas en el tiempo, entre las medias de los porcentajes de $\mathrm{CO}_{2}$, dependiendo del recubrimiento utilizado $(p<0,05)$. Mediante la evaluación gráfica, se observa que T7 presentó menores cambios, iniciando con $9,8 \%$ y alcanzando $11 \%$, a diferencia de T5, que alcanzó $14,13 \%$, que se atribuye a la permeabilidad al oxígeno de las películas con aceites esenciales, la cual, aumenta a medida que se incrementa la concentración de aceite en la formulación, considerando que las moléculas de $\mathrm{O}_{2}$ son solubles en lípidos; dichas películas pueden presentar canales hidrofóbicos, que permiten la difusión de gases.

En cuanto a los recubrimientos con almidón modificado de yuca, los tratamientos T4 y T2 presentaron menores cambios con porcentajes de $\mathrm{CO}_{2}$, de 11,3 hasta $15 \%$ y de 7,62 hasta $10,7 \%$, respectivamente, indicando que el almidón reduce la permeabilidad al $\mathrm{CO}_{2}$, debido, posiblemente, a la formación progresiva de zonas cristalinas, que actúan como barrera al paso del oxígeno, lo que también se observó en pimentones recubiertos con almidón y con almidón más aceite esencial de tomillo, siendo menor frente a aquellos que no fueron recubiertos (Ordoñez et al. 2014).

Firmeza. No se encontraron diferencias estadísticamente significativas entre medias de tratamientos para la firmeza $(p>0,05)$, pero sí entre puntos en el tiempo, indicando que 


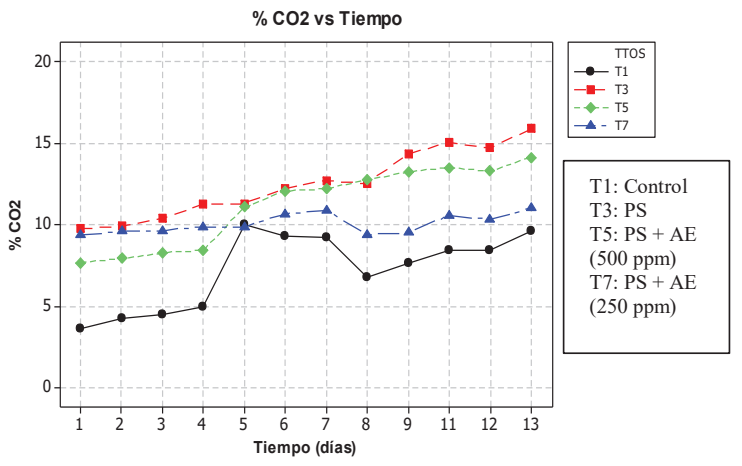

a.

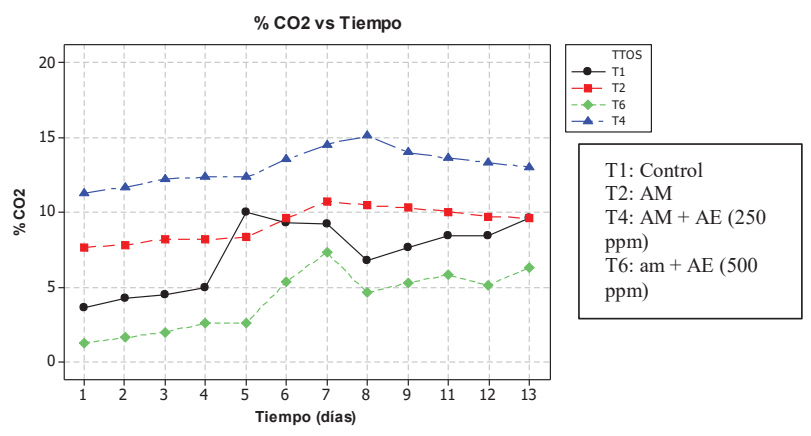

b.

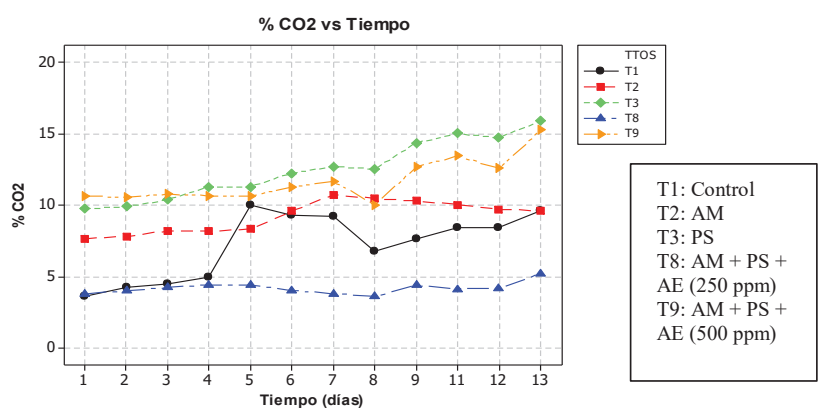

Figura 3. Porcentaje de $\mathrm{CO}_{2}$ respecto al tiempo. a) Tratamientos a base de proteína aislada de soya con y sin aceite esencial de orégano; b) Tratamientos a base de almidón modificado de yuca con y sin aceite esencial de orégano y c) Tratamientos que incorporan proteína aislada de soya, almidón modificado de yuca y aceite esencial de orégano.

el tiempo incide en los cambios de firmeza presentados. Se observó que los mayores cambios producidos fueron entre el día 1 , con un promedio de $12,39 \mathrm{~N}$ y el día 7 , con $4,45 \mathrm{~N}$ y los menores cambios, entre el día 7 y el 13, presentándose, en este último, el menor valor de 2,47N, los cuales, se atribuyen a la maduración y no a los tratamientos, al ser producidos por la acción de pectinasas y celulasas, que degradan las sustancias pécticas y la hemicelulosa, que se encuentran en la piel, debilitando las paredes celulares y las fuerzas cohesivas, que mantienen las células unidas (Hernández et al. 2010; Pinzón et al. 2007), además de la acción de hidrolasas, que contribuyen al ablandamiento (Barrera et al. 2012).

La firmeza presentó los siguientes valores de media y error estándar en los días, 1, 4, 7, 10 y 13, respectivamente: 12,3888 y 0,$71005 ; 9,0338$ y 0,$78475 ; 4,446$ y 0,54424 ; 4,0911 у 0,$54424 ; 2,4711$ у 0,28877 .

Sólidos solubles totales. Se observó un aumento progresivo en el contenido de sólidos solubles en todos los tratamientos, desde 9,77 hasta $11,53^{\circ}$ Brix, en promedio. Se aplicó una prueba de comparaciones múltiples robusta (Games-HoweII), que no detectó diferencias estadísticamente significativas entre los tratamientos ( $p>0,05)$, pero sí entre el tiempo de seguimiento, indicando que fue el tiempo el factor que influyó sobre las papayas.
En el día 1, se presentaron los valores más bajos, que se fueron incrementando con el tiempo. Entre los días 4 y 7, los valores fueron similares y luego aumentaron, progresivamente, en los días 10 y 13 (Tabla 2), indicando que los frutos continuaron su proceso de maduración después de alcanzar el pico climatérico (día 5); por otro lado, en el último día fueron más similares entre tratamientos (11 y $12^{\circ}$ Brix), evidenciando el proceso de senescencia, en donde los cambios en el fruto, se redujeron. En estudios similares, recubrimientos a base de almidón de yuca con aceites esenciales y proteína de soya, no afectaron el contenido en sólidos solubles y acidez (Saavedra \& Algecira, 2010).

pH. No hubo diferencias estadísticamente significativas entre los tratamientos ( $p>0,05)$, pero sí entre el tiempo de seguimiento, indicando que el tiempo influyó en los cambios de $\mathrm{pH}$ en las papayas (Tabla 2). Las diferencias indican un aumento de los valores, a causa de la disminución en el contenido de ácidos orgánicos, que son convertidos en azúcares, a medida que avanza la maduración (Hernández et al. 2010). Resultados similares fueron reportados por Andrade et al. (2014), sin diferencias significativas, al utilizar almidón de yuca y cera de laurel en combinación con aceite de oliva, en frutos de tomate de árbol, lo que demuestra que los recubrimientos, a partir de almidón, no inciden sobre esta variable. 
Tabla 2. Diferencias estadísticamente significativas para sólidos solubles, pH y porcentaje de acidez.

\begin{tabular}{|c|c|c|}
\hline \multicolumn{3}{|c|}{ Promedios de s.s en el tiempo } \\
\hline Tiempo & Media & Error estándar \\
\hline 1 día & $9,7152(\mathrm{a})^{*}$ & 0,14041 \\
\hline 4 días & $10,0474(\mathrm{a}) *(\mathrm{~b}) *$ & 0,09397 \\
\hline 7 días & $10,4085(b)^{*}$ & 0,09197 \\
\hline 10 días & $10,9248(\mathrm{c})^{*}$ & 0,06194 \\
\hline 13 días & $11,5319(d) *$ & 0,04383 \\
\hline \multicolumn{3}{|c|}{ PROMEDIOS DE ph EN EL TIEMPO } \\
\hline Tiempo & Media & Error estándar \\
\hline 1 días & $5,2519(a) *$ & 0,01703 \\
\hline 4 días & $5,3944(b)^{*}$ & 0,01794 \\
\hline 7 días & $5,3878(b)^{*}$ & 0,02746 \\
\hline 10 días & $5,5763(\mathrm{c})^{*}$ & 0,02734 \\
\hline 13 días & $5,5056(d) *$ & 0,02302 \\
\hline \multicolumn{3}{|c|}{ Promedios de \% acidez en el tiempo } \\
\hline Tiempo & Media & Error estándar \\
\hline 1 días & $0,0986(a)^{*}$ & 0,00341 \\
\hline 4 días & $0,0911(a)^{*}$ & 0,00412 \\
\hline 7 días & $0,1008(a)^{*}$ & 0,00371 \\
\hline 10 días & $0,0971(a)^{*}$ & 0,00379 \\
\hline 13 días & $0,1129(b)^{*}$ & 0,00425 \\
\hline
\end{tabular}

*Promedios de sólidos solubles, $\mathrm{pH}$ y porcentaje de acidez, con las mismas letras indican que no hay diferencias significativas $(\mathrm{p}>0,05)$ y promedios de sólidos solubles, $\mathrm{pH}$ y porcentaje de acidez, con letras diferentes, indican diferencias significativas $(\mathrm{p}<0,05)$.

Porcentaje de acidez. El Anova arrojó diferencias significativas $(p<0,05)$ en el tiempo de evaluación, pero no entre tratamientos, por lo que se aplicó la prueba Tukey, arrojando diferencias (Tabla 2).

Se registró un aumentó leve del porcentaje de acidez al final de la evaluación, debido a que los tratamientos T2 hasta T9, entre los días 10 y 13, aumentaron sus porcentajes, posiblemente, por el retraso en la volatilización de ácidos orgánicos, permitiendo que los frutos permanecieran más ácidos o ralentizaran la síntesis de ácidos orgánicos, debido a la alteración de la respiración en la producción de $\mathrm{CO}_{2}$ (Miranda et al. 2014); por el contrario, en los frutos control (T1), el porcentaje de acidez disminuyó, a partir del día 7, debido a la reducción de la actividad metabólica, porque los ácidos orgánicos usados como sustrato de la respiración, se agotaron durante este periodo (Miranda et al. 2014).
Crecimiento microbiano. Los resultados mostraron mayor daño en los frutos control (T1), con un área infectada mayor al 50\%, seguido por los tratamientos T2, T3, T5, T7, T8 y $\mathrm{T} 9$, con $22 \%$; los tratamientos que mejor se comportaron fueron T4 y T6, al reducir el porcentaje de daño, hasta $3 \%$. Se encontró que el aceite esencial, al ser mezclado con almidón, tuvo un efecto positivo sobre los frutos, ya que el almidón puede interferir en la maduración de los frutos (De Oliveira et al. 2016) y hacerlos menos susceptibles al ataque de patógenos (Kader, 2002); por otro lado, el aceite aporta sustancias, como timol, carvacrol, alfa terpineno y $p$-cimeno (Gómez \& López, 2009), metabolitos con propiedades antimicrobianas contra microorganismos fitopatógenos (Bosquez et al. 2010), al sumergir papayas en un recubrimiento con aceite esencial de tomillo, que tuvo efecto sobre el daño causado por los hongos Colletotrichum gloeosporioides y Rhizopus stolonifer. 
Según lo anterior, se observó que todos los recubrimientos lograron reducir la transpiración de las papayas y que la adición de aceite esencial a la matriz de almidón, a una concentración de 250 ppm, obtuvo los valores más bajos de las coordenadas L*, a* y b*, al mantener el color verde en los frutos, también un mejor control de la respiración, logrando reducir, considerablemente, los daños causados por el hongo Fusarium solani. No se observó efecto por parte de los recubrimientos sobre variables, como $\mathrm{pH}$, sólidos solubles y firmeza, cuyos cambios se debieron a la maduración inherente de los frutos.

Agradecimientos: Los autores expresan agradecimiento a la Universidad del Cauca, por los recursos dispuestos y facilitados para el desarrollo de la presente investigación. Conflicto de intereses: El manuscrito fue preparado y revisado con la participación de todos los autores, quienes declaran que no existe ningún conflicto de intereses, que ponga en riesgo la validez de los resultados presentados. Financiación: Este estudio fue financiado por la Universidad del Cauca de Popayán (Cauca, Colombia).

\section{BIBLIOGRAFÍA}

1. ADEME, A.; AYALEW, A.; WOLDETSADIK, K. 2013. Evaluation of antifungal activity of plant extracts against papaya anthracnose (Colletotrichum gloeosporioides). J. Plant Pathology \& Microbiology (Unites States of America). 4(10):1-4.

2. ANDRADE, J.; ACOSTA, D.L.; BUCHELI, M.A.; OSORIO, O. 2014. Desarrollo de un recubrimiento comestible compuesto para la conservación del tomate de árbol (Cyphomandra betacea S.). Información tecnológica (Chile). 25(6):57-66.

3. BARNETT, H.L.; HUNTER, B.B. 1998. Illustrated genera of imperfect fungi. Ed. The American Phytopathological Society (United States of America). 240p.

4. BARRERA, E.; GIL, M.; GARCÍA, C.M.; DURANGO, D.L. 2012. Empleo de un recubrimiento formulado con propóleos para el manejo poscosecha de frutos de papaya (Carica papaya L. cv. Hawaiana). Rev. Facultad Nacional de Agronomía (Colombia). 65(1):64976506.

5. BOSQUEZ, E.; RONQUILLO, E.; BAUTISTA, S.; VERDE, J.R.; MORALES, J. 2010. Inhibitory effect of essential oils against Colletotrichum gloeosporioides and Rhizopus stolonifer in stored papaya fruit and their possible application in coatings. Postharvest biology and technology (United States of America). 57(2):132-137.
6. CASTRICINI, A.A.; CAVESTRÉ, R.C.; DELIZA, R. 2012. Starch edible coating of papaya: effect on sensory characteristics. Food Science and Technology (Brasil). 32(1):84-92.

7. DE ANCOS, B.; GONZÁLEZ, D.; COLINA, C.; SÁNCHEZ, C. 2015. Uso de películas / recubrimientos comestibles en los productos de iv y v gama. Rev. Iber. Tecnología Postcosecha (España). 16(1):8-17.

8. DE OLIVEIRA, B.F.; CRUZ, A.F.; ALVES, E. 2016. Cassava starch coatings for postharvest control of papaya anthracnose. Phytopathologya Mediterranea (Italy). 55(2):276-284.

9. FERNÁNDEZ, D.; BAUTISTA, S.; FERNÁNDEZ, D.; OCAMPO, A.; GARCÍA, A.; FALCÓN, A. 2015. Películas y recubrimientos comestibles: una alternativa favorable en la conservación poscosecha de frutas y hortalizas. Rev. Ciencias Técnicas Agropecuarias (Cuba). 24(3):52-57.

10. GÓMEZ, A.I.; LÓPEZ, A. 2009. Potencial antimicrobiano de los aceites esenciales de orégano (Origanum vulgare) y canela (Cinnamomum zeylanicum). Temas selectos de ingeniería de alimentos (México). 13:3345.

11. GUÉDEZ, C.; CAÑIZALEZ, L.; AVENDAÑO, L.; SCORZA, J.; CASTILLO, C.; OLIVAR, R.; MÉNDEZ, Y.; SÁNCHEZ, L. 2014. Actividad antifúngica del aceite esencial de naranja (Citrus sinensis L.) sobre hongos postcosecha en frutos de lechosa (Carica papaya L). Revista de la Sociedad Venezolana de Microbiología (Venezuela). 34:81-85.

12. GUERREIRO, A.; GAGO, C.; FALEIRO, M.; MIGUEL, M.; ANTUNES, M. 2015. The effect of alginate-based edible coatings enriched with essential oils constituents on Arbutus unedo L. fresh fruit storage. Postharvest Biology and Technology (Portugal). 100:226-233.

13. FIGUEROA, J.; SALCEDO, J.; NARVÁEZ, G. 2013. Efecto de recubrimientos comestibles a base de almidón nativo y oxidado de yuca sobre la calidad de mango (Tommy Atkins). Temas agrarios (Colombia). 18(2): 94-105.

14. HERNÁNDEZ, M.S.; BARRERA, J.; MELGAREJO, L.M. 2010. Fisiología poscosecha. En: Experimentos en fisiología vegetal. Ed. Universidad Nacional de Colombia (Colombia). p.167-186. 
15. KADER, A.A. 2002. Postharvest biology and technology: an overview. En: Postharvest technology of horticultural crops. Ed. University of California (United States of America). p.39-48.

16. MIRANDA, A.D.; ALVIS, A.; ARRAZOLA, G. 2014. Efectos de dos recubrimientos sobre la calidad de la papaya (Carica papaya) variedad tainung. Temas agrarios (Colombia). 19(1):7-18.

17. NAVARRO, J.R.; ARAUZ, L.F. 1999. Exactitud y repetibilidad de dos métodos para la evaluación de la severidad de enfermedades fungosas en el fruto de la papaya (Carica papaya). Agronomía Costarricense (Costa Rica). 23(1):89-96.

18. OCORÓ, M.; AYALA, A. 2013. Influencia del espesor en secado de puré de papaya (Carica papaya l.) por tecnología de ventana de refractancia. Dyna (Colombia). 182:147-154.

19. ORDOÑEZ, D.Y.; ZUÑIGA, D.; HOYOS, J.L.; MOSQUERA, S.A.; MOSQUERA, L.P. 2014. Efecto de recubrimiento de almidón de yuca modificado y aceite de tomillo aplicado al pimiento (Capsicum annuum). Revista Mexicana de Ciencias Agrícolas (México). 5(5):795-805.

20. PAZ, S.P.; MOSQUERA, S.A.; VILLADA, H.S. 2016. Evaluación de la permeabilidad de un recubrimiento elaborado a partir de almidón de yuca nativo y modificado. Vitae (Colombia). 23(supl 1):s222-s225.

21. PINZÓN, I.M.P.; FISCHER, G.; CORREDOR, G. 2007. Determinación de los estados de madurez del fruto de la gulupa (Passiflora edulis Sims.). Agronomía Colombiana (Colombia). 25(1):83-95.
22. QUINTERO, C.J.; FALGUERA, V.; MUÑOZ, A. 2010. Películas y recubrimientos comestibles: importancia y tendencias recientes en la cadena hortofrutícola. Tumbaga (Colombia). 1(5):93-118.

23. SAAVEDRA, N.; ALGECIRA, N. 2010. Evaluación de películas comestibles de almidón de yuca y proteína aislada de soya en la conservación de fresas. NOVA (Colombia). 8(14):171-182.

24. SANTAMARÍA, F.; SAURI, E.; ESPADAS, F.; GIL, R. 2009. Postharvest ripening and madurity indices for Maradol papaya. Interciencia (México). 24(8):583-588.

25. SIQUEIRA, C.; MACHADO, M.; NASCIMENTO, A.; RODRIGUES, M. 2012. Control of Papaya Fruits Anthracnose by Essential Oil of Ricinus communis. Brazilian Archives of Biology and Technology (Brazil). 55(1).75-80.

26. VÁZQUEZ, M.C.; GUERRERO, J.A. 2013. Recubrimientos de frutas con biopelículas. Temas Selectos de Ingeniería de Alimentos (México). 7(2):5-14.

27. VILLA, A.; PÉREZ, R.; MORALES, H.A.; BASURTO, M.; SOTO, J.M.; MARTÍNEZ, E. 2015. Situación actual en el control de Fusarium spp. y evaluación de la actividad antifúngica de extractos vegetales. Acta Agronómica (Colombia). 64(2):194-205.

Recibido: Enero 6 de 2018

Aceptado: Abril 13 de2018

Cómo citar:

Castillo Silva, D.A.; Salazar Anacona, K.; Mosquera Sánchez, S.A.; Rengifo Canizales, E. 2018. Efecto de recubrimientos de almidón modificado de yuca, proteina aislada de soya y aceite esencial de orégano aplicados a la papaya. Rev. U.D.C.A. Act. \& Div. Cient.21(1): 71-80. 